\title{
Impairment of Continuous Insulin Delivery Therapy and Analysis from Graeco-Latin Square Design Model
}

\author{
Norou Diawara1, Ayodeji Demuren², Eric Gyuricsko ${ }^{3}$ \\ ${ }^{1}$ Department of Mathematics and Statistics, Old Dominion University, Norfolk, VA, USA \\ ${ }^{2}$ Department of Mechanical and Aerospace Engineering, Old Dominion University, Norfolk, VA, USA \\ ${ }^{3}$ Department of Pediatrics, Eastern Virginia Medical School, Norfolk, VA, USA \\ Email: ndiawara@odu.edu
}

Received 15 July 2016; accepted 14 August 2016; published 17 August 2016

Copyright (C) 2016 by authors and Scientific Research Publishing Inc.

This work is licensed under the Creative Commons Attribution International License (CC BY). http://creativecommons.org/licenses/by/4.0/

(c) (i) Open Access

\section{Abstract}

The desire to deliver measured amount of insulin continuously to patients with type I diabetes, for glycemic control, has attracted a lot of attention. Continuous subcutaneous insulin infusion has seen some success in recent years. However, occlusion of insulin delivery may prevent the patient from receiving the prescribed dosage, with adverse consequence. An in vitro study of insulin delivery is performed, using different insulin pumps, insulin analogs and operating conditions. The aim is to identify incidences of occlusion due to bubble formation in the infusion line. A detailed statistical analysis was performed on the data collected to determine any significant differences and deviations in insulin delivery rates that might be due to factors such as: pump type, the set basal flow rate, insulin type, vibration, and possible insulin occlusion due to air bubble formation within the infusion line. Our findings from the Graeco-Latin Square design model show that there are statistical differences due to the devices, and statistical identifiable clusters are used to distinguish the devices. Such hierarchical models used to describe the analyses, include the flow rate, the pump types, and the activity level.

\section{Keywords}

Graeco-Latin Square Design, Insulin Delivery, Model Based Cluster Analysis, Occlusion

\section{Introduction}

Type 1 diabetes affects about 1 in 400 children under the age of 18 years. Continuous subcutaneous insulin infu-

How to cite this paper: Diawara, N., Demuren, A. and Gyuricsko, E. (2016) Impairment of Continuous Insulin Delivery Therapy and Analysis from Graeco-Latin Square Design Model. Journal of Biosciences and Medicines, 4, 40-51. 
sion (CSII), also known as insulin pump therapy, is now commonplace [1] [2], even in the care of the youngest persons (i.e., under 5 years old) with Type 1 diabetes [3]. CSII tries to deliver insulin to the patient on a continuous basis similar to the natural internal secretion of insulin from the pancreas [4]. However, our findings below show that the process is intermittent, rather than continuous. During CSII, insulin must travel from the pump's reservoir through the infusion set, which consists of a length of capillary tubing connected to a catheter, to the patient's subcutaneous space without occlusion. This must occur accurately, regardless of ambient temperature, activity level of the patient, or basal dosage rate. The typical basal dosage rate for adults is about 10 micro-liters (or 1 unit (U) of insulin) per hour, whereas in infants it is typically much less (about 1 micro-liter or 0.1 unit (U) of insulin per hour). The basal dosage may be supplemented with boosters called bolus insulin dosage, as needed, before meals. As a consequence of this low basal infusion rate, occlusion to insulin flow can, and often does occur. Occlusion of insulin flow is a potentially dangerous, unforeseen circumstance that places the patient with diabetes using CSII at imminent risk, if not detected and immediately treated. Severe, sustained hyperglycemia or diabetic ketoacidosis (DKA) may result [5]. Frequent occlusive episodes may result in suboptimal glycemic control, elevated hemoglobin A1C levels and an increased risk of long-term complications from diabetes [6]. Anecdotal reports and a few studies have examined causes of occlusions in CSII, concluding that insulin type may be a contributor [7] [8]. Poulsen et al. [9] suggested that infection at the infusion site or some physiological reason could be responsible for insulin occlusion in CSII patients. Demuren and Doane (2007) [10] performed in vitro studies to simulate CSII and observed insulin occlusion through bubble formation. Kerr et al. (2008) [11] performed insulin occlusion studies at low flow rates, but the emphasis was on complete occlusion which triggered pump alarm. On the contrary, occlusion due to bubble formation in infusion set is temporary and would not trigger the pump alarm. However, the effect could be just as detrimental for glycemic control, since it was observed [10] that insulin delivery could be interrupted for up to 10 hours.

The present paper reports on a parametric in vitro study, performed to simulate CSII under varying conditions of pump type, basal dosage rate, activity level, etc. Although this controlled laboratory study does not use real test subjects and therefore cannot address effects of possible insulin occlusion due to physiological interactions between insulin, infusion set and the patient, other more basic factors are investigated. The paper is organized as follows. In Section 2, the nature of the data is discussed, and a need for data model characterization is presented. Section 3 specifies the model for Graeco-Latin Square and its use for statistical inference. Our proposed approach is based on practicality and is pragmatic, seeking to identify differences based on the devices. The effects of the selected factors are studied. In Section 4, the results are presented. Section 5 contains a brief summary.

\section{The Experimental Setup and the Nature of Data}

The experimental setup is designed to reproduce typical operating conditions for CSII. Figure 1 shows a schematic of the experimental setup. Three flow lines are investigated simultaneously. Three insulin pumps are attached to a temperature controlled hot water jacket to simulate body temperature and are placed on an IKA KS control orbital shaker table which can be programmed to simulate various levels of accelerations to mimic intensity of physical activity of the patients with diabetes. Insulin pump reservoirs are filled with desired insulin and primed to eliminate all bubbles from the infusion set.

Since the pump is usually close to the patient's body, the model can assume that temperature is fixed. So a single temperature setting will be used to obtain information, and data will be used in conjunction with this to determine significant difference between the values of the dependent variables.

Given a fixed time periods, the parameters can be estimated by the maximum likelihood method. The issue is that in most applications, the Gaussian mixtures formalize the shape of the flow distribution. However, the significant difference between the insulin types, the types of pumps, the activity levels are not known, and little to no data on them has been recorded.

The desired basal rate is set and each experiment is run for 5 day duration (hereafter denoted as week), as is commonly used in CSII therapy. Volume flow rates of insulin through the CSII infusion sets are measured with Sensirion SLG1430-480 flow meters and recorded on a computer every 0.64 second, along with pump temperatures and total mass of insulin deposited in bottles placed on analytical balances (Sartorius TE-64) with sensitivity of $0.0001 \mathrm{~g}$. The overall total volume of insulin, which has been pumped over a particular period, is obtained by integrating the recorded flow meter readings. This can then be compared, after multiplying with insulin density, 
to the recorded analytical balance reading. Thus, the flow meters indicate instantaneous flow rate, whereas the scales record cumulative flow. There is considerable lag in the times both instruments record the flow, since at the very low flow rates it may take several hours for insulin to traverse the length of the infusion line, from the Sensiron flow meter to the collection bottle on the scale. However, as shown in Figure 2, over long periods, the slope of the volume versus time curves should be the same, which validates consistency of measuring instruments. It should be noted that the flow of insulin is highly intermittent and so is very difficult to measure. Rather

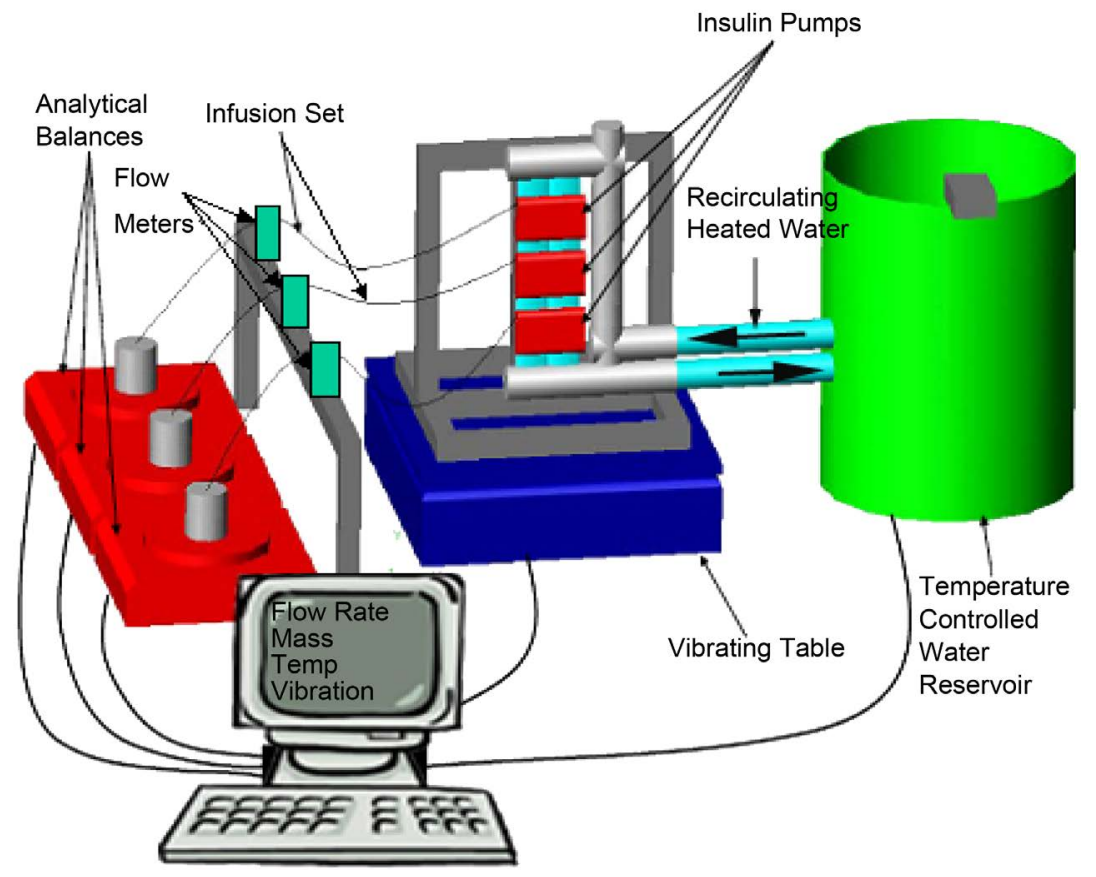

Figure 1. Sketch of the experimental setup.
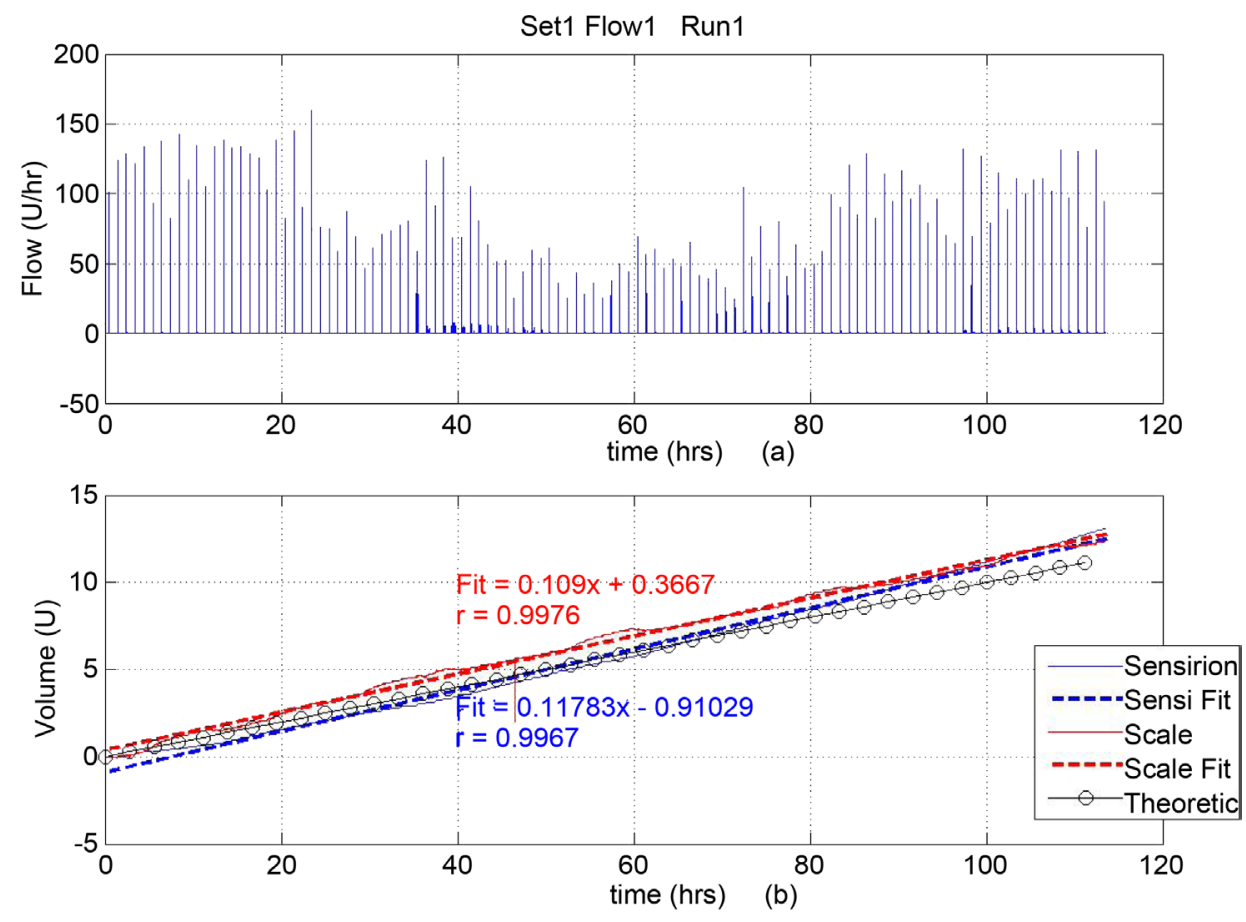

Figure 2. Set 1 Flow 1 Run 1: 0.1 U/hr, M511 \#1, Humalog, 0-RPM (a) Flow rate, (b) Volume. 
than operate continuously, insulin pumps move the fluid pulsatingly. Demuren and Doane (2007) [10], and Wang et al. (2011) [12] have investigated details of the operation of the Medtronic M511 pump. Demuren and Doane [10] observed that for the nominal flow rate of $0.1 \mathrm{U} / \mathrm{hr}$, there was only a single pulse of the pump in the hour, with total duration of about 0.25 seconds to move the $0.1 \mathrm{U}$. This pulse is repeated at suitable intervals to achieve higher flow rates, e.g., every 20 minutes to achieve $0.3 \mathrm{U} / \mathrm{hr}$ and every 6 minutes for $1 \mathrm{U} / \mathrm{hr}$. Wang et al. [12] used sophisticated micro-PIV (Particle Image Velocimetry) to study the resulting flow velocities in detail. They observed that each pump pulse lasted for 0.4 seconds, with the fluid velocity rising as high as $26.4 \mathrm{~mm} / \mathrm{s}$ at the center, or 260 times the calculated mean velocity. It subsequently decayed exponentially with a time constant of 0.16 seconds, such that very little flow can be discerned 3 seconds after each pulse. Other pump types operate in similar pulsating manner, but at different frequencies. It is a challenge to calibrate the Sensiron flow meters to record accurately such rapidly varying unsteady flow. The flow meters register presence and duration of air/vapor bubbles through gaps in reading.

Four different models of insulin pumps are investigated, namely, the Paradigm M511, Paradigm M712, and Animas IR-1200. By design, the pumps operate intermittently at a frequency, which depends on the set basal rate. For example, the Paradigm M511 delivers $0.1 \mathrm{U}$ per actuation in the basal dosage mode. At a basal rate of $0.1 \mathrm{U} / \mathrm{hr}$, the pump would be actuated once per hour, at $0.3 \mathrm{U} / \mathrm{hr}$ three times per hour, at $1.0 \mathrm{U} / \mathrm{hr}$ ten times per hour, and so on. The pumps are connected to the flow meters and collection bottles, placed on the analytical balances, via two types of infusion sets, namely, the Sof-Set (0.48 $\mathrm{mm}$ i.d.) and the Quick Set $(0.36 \mathrm{~mm}$ i.d.). These are standard infusion sets used in practice, and have lengths of between 20 and $40 \mathrm{~cm}$. The catheter, at the end of the infusion set, is sealed to the collection bottle to prevent any evaporation over the five day period of the experiments. The three types of insulin used in this study: Apidra (glulisine), Humalog (lispro), and Novolog (aspart), are rapid acting insulin analogs in common use [13] [14].

The Sensirion flow meter can measure a maximum flow rate of 40,000 $\mathrm{nl} / \mathrm{min}(240 \mathrm{U} / \mathrm{hr})$ with a resolution of $7 \mathrm{nl} / \mathrm{min}(0.042 \mathrm{U} / \mathrm{hr})$ and a repeatability of $0.6 \%$ of the measured value. The flow meter is very sensitive to environmental conditions and must be recalibrated frequently. For this purpose, the Harvard PHD 2000 Programmable calibration apparatus was utilized. The calibration is performed to ensure that the Sensiron flow meter can accurately record flow rates in the range between $1 \mathrm{U} / \mathrm{hr}$ and $120 \mathrm{U} / \mathrm{hr}$.

\section{Graeco-Latin Square Design and Statistical Model}

The objective of this study is to assess and compare the performance of the insulin delivery based on the type of pumps, the level of activity characterized by the intensity of vibration, the insulin type, and the flow rate level. The study of interaction will assess the understanding of the structure and modeled insulin delivery. The simulations are carried out to illustrate the behavior of the process. Since each of the experiments takes five days to run, the number of replicates is typically small, and would lead to low statistical power and large variance estimates [15] [16]. As a remedy to those facts, a design of experiments has been carefully selected.

In so doing, we aim for simulation, optimization and prediction under a sound experimental design. We propose a four-way Graeco-Latin squares design. The analysis provides a theoretical basis for the comparison of flow rates, insulin types (Humolog, Apidra, Novolog), pump types (M511-\#1, M511-\#2, M511-\#3, M712, Animas), vibrations, and flow meters (line 1, 2 or 3 ) without ignoring noise dependence. The flow rates are set on low to $0.1 \mathrm{U} / \mathrm{hr}$, medium to $0.3 \mathrm{U} / \mathrm{hr}$, and high to $1.0 \mathrm{U} / \mathrm{hr}$. The vibration levels are set on low to 0 , medium to 100 and high to 200 RPM. The design is chosen in such a way that test of the difference between factors can be made without having to run each of the $34=81$ cases individually [17] [18]. Running the 81 possible cases may not be realistic, because of financial and time limitations. The Graeco Latin square design is built to yield a larger optimal information gathering, taking into account the fact that each data set cluster takes approximately one week to collect. It is very efficient since it can block 3 nuisance factors: flow rate, insulin type, pump type, and vibrations. The temperature has been set fixed, at a nominal value of 90 degrees Fahrenheit. Table 1 shows the experimental design combination from the four factors. As described, each pair of treatments appears once in the grid. Fifteen configurations are selected as suggested in this design. In each week, three of the experiments are run under the three distinct lines. Experiments are repeated in a further week, as signified by " 2 runs" in the table, to confirm the results.

To formulate the problem, the standard linear equation model for a Graeco-Latin Square design without interaction effects is as follows: 
Table 1. Experimental configuration.

\begin{tabular}{|c|c|c|c|c|}
\hline & & Line 1 & Line 2 & Line 3 \\
\hline Week 1 & Flow rate & $0.1 \mathrm{U} / \mathrm{hr}$ & $0.3 \mathrm{U} / \mathrm{hr}$ & $1.0 \mathrm{U} / \mathrm{hr}$ \\
\hline \multirow[t]{3}{*}{2 runs } & Insulin & Humalog & Apidra & Apidra \\
\hline & Pump & M511-\#1 & M511-\#2 & Animas \\
\hline & Vibration & Vib1 (0 RPM) & Vib1 (0 RPM) & Vib1 (0 RPM) \\
\hline Week 2 & Flow rate & $0.1 \mathrm{U} / \mathrm{hr}$ & $0.3 \mathrm{U} / \mathrm{hr}$ & $1.0 \mathrm{U} / \mathrm{hr}$ \\
\hline \multirow[t]{3}{*}{2 runs } & Insulin & Novolog & Apidra & Novolog \\
\hline & Pump & Animas & M511-\#1 & M511-\#3 \\
\hline & Vibration & Vib2 (100 RPM) & Vib2 (100 RPM) & Vib2 (100 RPM) \\
\hline Week 3 & Flow rate & $0.3 \mathrm{U} / \mathrm{hr}$ & $0.3 \mathrm{U} / \mathrm{hr}$ & $1.0 \mathrm{U} / \mathrm{hr}$ \\
\hline \multirow[t]{3}{*}{2 runs } & Insulin & Humalog & Humalog & Novolog \\
\hline & Pump & M511-\#3 & Animas & M511-\#1 \\
\hline & Vibration & Vib3 (200 RPM) & Vib3 (200 RPM) & Vib3 (200 RPM) \\
\hline Week 4 & Flow rate & $0.3 \mathrm{U} / \mathrm{hr}$ & $0.1 \mathrm{U} / \mathrm{hr}$ & $0.3 \mathrm{U} / \mathrm{hr}$ \\
\hline \multirow[t]{3}{*}{2 runs } & Insulin & Apidra & Apidra & Novolog \\
\hline & Pump & M511-\#2 & M511-\#3 & M712 \\
\hline & Vibration & Vib1 (0 RPM) & Vib1 (0 RPM) & Vib1 (0 RPM) \\
\hline Week 5 & Flow rate & $0.1 \mathrm{U} / \mathrm{hr}$ & $0.3 \mathrm{U} / \mathrm{hr}$ & $1.0 \mathrm{U} / \mathrm{hr}$ \\
\hline \multirow[t]{3}{*}{2 runs } & Insulin & Apidra & Novolog & Humalog \\
\hline & Pump & M712 & M511-\#3 & M712 \\
\hline & Vibration & Vib3 (200 RPM) & Vib2 (100 RPM) & Vib2 (100 RPM) \\
\hline
\end{tabular}

$$
Y_{i j k l}=\mu+\theta_{i}+\tau_{j}+\beta_{k}+w_{l}+e_{i j k l},
$$

where $Y_{i j k l}$ denotes the quantity of insulin delivery for the experimental unit with the $i^{\text {th }}$ flow rate, the $j^{\text {th }}$ insulin type, the $k^{\text {th }}$ pump type, and the $l^{\text {th }}$ vibration level, $\mu$ is the overall mean, $\theta_{i}$ is the flow rate effect, $\tau_{j}$ is the insulin type effect, $\beta_{k}$ is the pump type effect, $w_{l}$ is the vibration effect and $e_{i j k l}$ is the random error, with $i, j, k, l=1,2,3$.

As usual, the effects are subject to the restriction that:, $\sum_{i=1}^{3} \theta_{i}=\sum_{j=1}^{3} \tau_{j}=\sum_{k=1}^{3} \beta_{k}=\sum_{l=1}^{3} w_{l}=0$ and the errors are independent $N\left(0, \sigma^{2}\right)$.

Flow data of the insulin delivery can provide important information about the differences in the insulin types, pump types, and dynamic circuitries. These counts are correlated and over disperse. There is no straightforward method of collecting such data than setting up the calibration before each experiment. In fact, the Graeco-Latin square design turns out to be the most suitable for this experiment with three levels of the flow rate, pump types, vibration level. It is at the cornerstone for evaluating new interventions and will allow us to solve challenging clinical trial set up [19]. Preliminary results show that there could be a general shift in outcome since the desired flow settings were not always met in practice. The outlier values were removed since they led to higher than acceptable variance tolerance. As the simulations reveal, the latter is by no means exceptional. A cut off value formalizes the separation between clusters and is guided by data from borderline distributions. Some experiments are also run twice to reduce the error, and increase accuracy in the model design.

\section{Simulation Results}

The data represent thousands of values of time and flow quantities recorded over a period of five days. Experimentations are one way of obtaining and analyzing data for such issues. Typical experimental data, collected for 
one of the runs, are shown in Figure 2. The mass data were measured by the analytical-balance-scale \#1 and the flow rate data were measured by the Sensirion flow meter \#1 for the Paradigm M511-\#1 pump, which was set to deliver $0.1 \mathrm{U} / \mathrm{hr}$ of Humalog insulin, for the experimental configuration Set 1 Flow 1 Run 1. Figure 1(a) shows the flow rate versus time as measured at a frequency of $1.5625 \mathrm{~Hz}$ or every 0.64 seconds. Based on the flow rate data the cumulative volume was calculated by taking the integral of the measured flow rate with respect to time in hour. The cumulative volume, based on the scale's measurement of total mass of insulin delivered, is also calculated from the total mass of insulin recorded approximately every 2 seconds, divided by the density of insulin. These two cumulative volume estimates, along with corresponding linear regression fits, are compared with the "theoretical" or ideal delivery volumes based on the nominal pump basal dose rate. Over the 115 hours ( $\sim 5$ days) of data collection, there is a good correlation between measured and expected volume flow rates. There is also no evidence of bubble formation. Hence, in this case, the pump operated as expected, delivering the set basal rate over the whole 5-day period.

All model analyses under Equation (1) are performed several times due to the weekly data collected, and compressed using SAS ${ }^{\circledR} 9.2$ on the 10 week experiments. Under the general linear model framework, the general class described in (1) was fitted. The analysis investigates first the effects of measurement errors on the estimations of the parameters of interest such as mean and variance. Ignoring measurement error can lead to inconsistent estimators. In that sense, we proposed a bias reduction by calibrating the instruments. A check of the normality of the data is not in our cases valid option. The insulin is delivered by influx in selected time periods. The data does not follow normal assumption settings. Nevertheless, it must be decreasing before another influx of insulin is delivered. Our first analysis showed that the delivery was not equally spread and consistent, and this causes attenuation in the estimators. This behavior is typical in all the instruments, and called attention for calibration. When a "gold" standard is available, a validation study can then be performed, and design of analysis can be used to test assumptions of data and significant difference among the factors.

The estimates of the parameters of the model of Equation (1) based on a sample of 1609 observation values are reported in Table 2.

The overall analysis of the model Equation (1) shows that all the effects are significant and must be entered in the model. So, the full model is significant. From Table 3 of the analysis of variance output, the predictors insulin, flow meter, flow rate, pump type and vibration, have an effect on the measured flow rate, with all p-values associated with the F-statistics for these variables very small, all less than 0.05 . So there is strong evidence that of a difference in the volume delivery based on the selected characteristics. We will next explore further the effect of each variable separately.

\subsection{Effect of Flow Rate}

Experiments were performed at three chosen levels of flow rate: $0.1,0.3$ and at $1.0(\mathrm{U} / \mathrm{hr})$. It was expected that

Table 2. Estimates of parameters of model.

\begin{tabular}{|c|c|c|c|c|c|c|}
\hline \multirow{2}{*}{$\begin{array}{l}\text { Overall mean } \\
\qquad \widehat{\mu}=0.432\end{array}$} & Flow rate & Insulin types & \multicolumn{2}{|c|}{ Pump Types } & \multicolumn{2}{|c|}{ Vibration level } \\
\hline & $\hat{\theta}_{1}=0.297 \quad$ Rate $0.1 \mathrm{U} / \mathrm{hr}$ & $\widehat{\tau_{1}}=-0.128 \quad$ (Apidra) & $\widehat{\beta}_{1}=0.270$ & Pump type M712 & $\widehat{w_{1}}=-0.151$ & No vibration \\
\hline & $\widehat{\theta}_{2}=-0.1404$ Rate $0.3 \mathrm{U} / \mathrm{hr}$ & $\widehat{\tau_{2}}=0.004$ (Humolog) & $\widehat{\beta}_{2}=-0.114$ & Pump type Animas & $\widehat{w_{2}}=0.132$ & $\mathrm{Vib}=100$ \\
\hline & $\widehat{\theta}_{3}=0.4234$ Rate $1.0 \mathrm{U} / \mathrm{hr}$ & $\widehat{\tau_{3}}=0.148 \quad$ (Novolg) & $\widehat{\beta_{3}}=-0.023$ & Pump type M511 & $\widehat{w_{3}}=0.084$ & $\mathrm{Vib}=200$ \\
\hline
\end{tabular}

Table 3. ANOVA output.

\begin{tabular}{cccccc}
\hline Source & Degree of Freedom & Type I Sum of Squares & Mean Sum of Squares & F Value & P-values \\
\hline Insulin & 2 & 21.356 & 10.678 & 191.87 & $<0.01$ \\
Flow rate & 2 & 120.986 & 60.493 & 1086.96 & $<0.01$ \\
Pump type & 2 & 5.014 & 2.507 & 45.05 & $<0.01$ \\
Vibration & 2 & 4.185 & 2.092 & 37.60 & $<0.01$ \\
\hline
\end{tabular}


lower flow rates will lead to lower insulin delivery, and that is exactly what was observed in the data as summarized in Table 4. Higher design flow rates yielded higher total volume and measured flow rates. In other words, the flow rate is a significant contributor of the insulin delivery. Such a result was much evidenced in week 4. In fact, the volume in week 4 suggests that there is significant difference between the flow rate $0.1,0.3$ and 1.0 $\mathrm{U} / \mathrm{hr}$, even if there is no vibration effect. For further comparisons, the Scheffe, Bonferroni and Tukey tests of significant difference were also computed. The results are consistent with the findings. One point to notice is the change in the standard deviation. This limitation may have affected the outcomes.

The panel plot of the flow rates 0.1, 0.3 and 1.0 for the insulin types is shown in Figure 3. At the design flow rate of $0.3(\mathrm{U} / \mathrm{hr})$, there are higher variations for both Apidra and Humolog. However, their effects on the average measured flow rate are not significant, since the mean turns out to be about the same as the designed flow rate.

Table 4. Summary of measured flow rate by designed flow rates and insulin types.

\begin{tabular}{cccc}
\hline Design Flow rate $(\mathrm{U} / \mathrm{hr})$ & Insulin Type & Measured Flow rate $(\mathrm{U} / \mathrm{hr})$ & Standard Deviation (U/hr) \\
\hline & Apidra & 0.155 & 0.026 \\
& Humalog & 0.115 & 0.030 \\
& Novolog & 0.133 & 0.014 \\
& Apidra & 0.274 & 0.324 \\
0.3 & Humalog & 0.278 & 0.018 \\
& Novolog & 0.374 & 0.085 \\
& Apidra & 0.553 & 0.028 \\
\hline
\end{tabular}

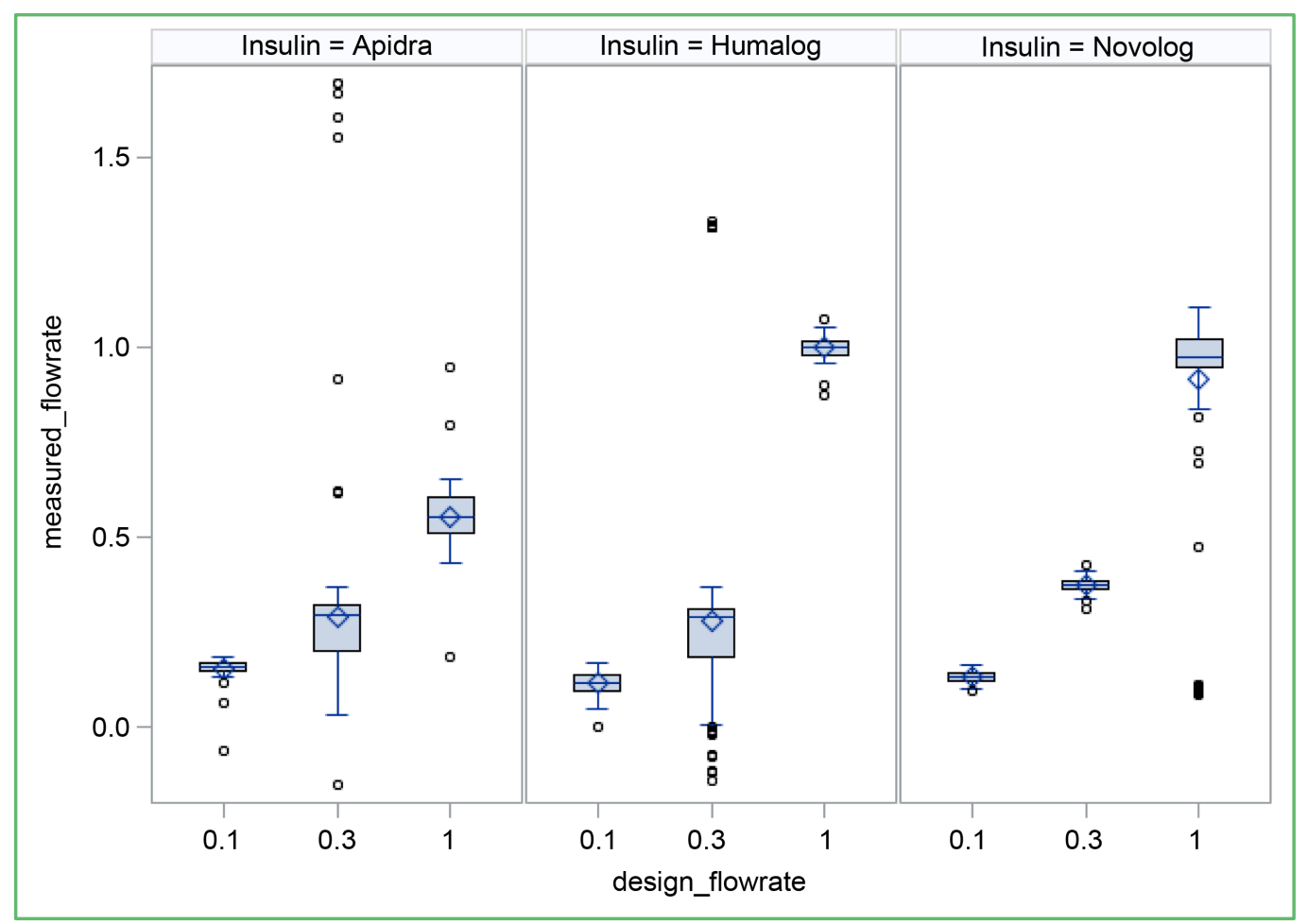

Figure 3. Design vs measured flow rates (U/hr) for different insulin types. 


\subsection{Effect of Insulin}

Three insulin types that were utilized; namely Humalog, Novolog and Apidra. The results of the measured flow rates for each of the insulin types are summarized in Table 4 and plotted in Figure 4. While Apidra is the insulin that has been used the most ( 6 times), followed by Novolog (5 times) and Humalog (4 times), there is clear distinction between the insulins. Novolog is the least stable at high flow rate. However, at low and medium flow rates, Novolog seems to have smaller variation associated with the CSII. Humalog performed well at low (0.1 $\mathrm{U} / \mathrm{hr}$ ) and high (1.0 U/hr) flow rates. Apidra performed worse at medium flow rate of $0.3 \mathrm{U} / \mathrm{hr}$. At all three flow rates, there is a significant difference between the three insulin types with p-values less than 0.05 level of significance. However, it was worth noting that at flow rate of $0.3 \mathrm{U} / \mathrm{hr}$, there were no significant difference between Humolog and Apidra, both displaying high variations.

\subsection{Effect of Pump Types}

There were three types of pumps: M511, M712, and Animas. Three pumps of type M511 were utilized, we call them M511-\#1, M511-\#2, and M511-\#3. Each pump was used three times or more except for M511-\#2 (2 times). Table 5 summarizes the measured flow rates for the different pump types and Figure 5 compares the data histogram with the different pump types. It is normally attached directly to the body, and it is difficult to simulate such operation appropriately in this study.

Animas has been used three times on week 1 through week 3 . It has been very consistent in its measured flow rates and means except on week 3, where the vibration level used was 200 RPM.

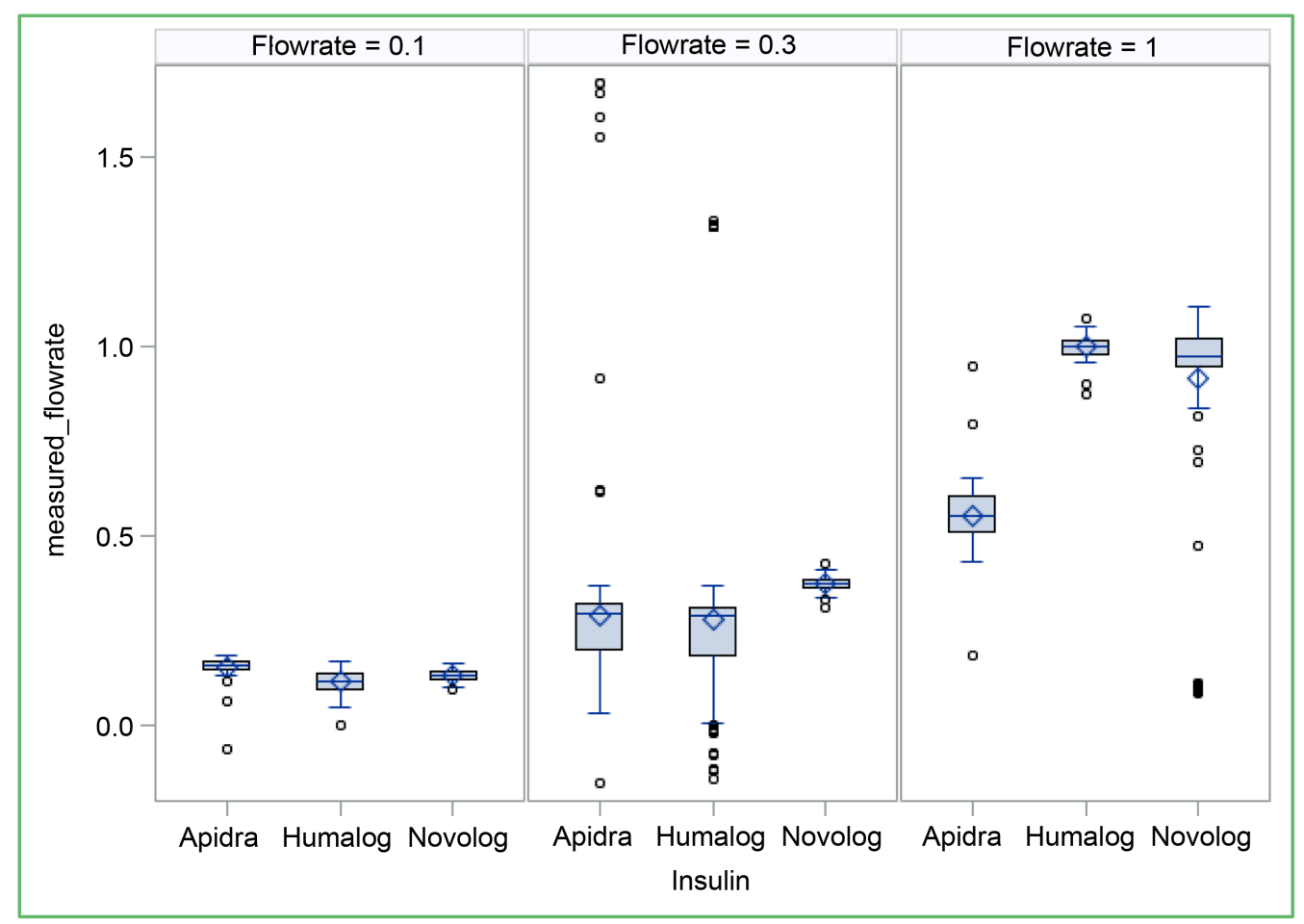

Figure 4. Summary of Insulin types at different flow rates (U/hr).

Table 5. Table of means and standard deviations of measures flow rates (U/hr) for each pump type.

\begin{tabular}{ccc}
\hline Pump type & Mean & Standard deviation \\
\hline M712 & 0.701 & 0.314 \\
Animas & 0.318 & 0.306 \\
M511 & 0.409 & 0.401 \\
\hline
\end{tabular}


Pump type M712 has been used at all levels of flow rate, and at different vibration levels. It has shown consistency in its results. The standard deviations recorded were quite reasonable. One thing to notice is that the high vibration was set at lower flow rate. This is a suggestive idea as to minimize variation in the flow for pump type M712, making it fit more accurately to the patient's need.

\subsection{Effect of Vibration}

The effects of the vibration level on measured flow rates are shown in Table 6. The analysis shows that there is significant difference in the outcomes for flow rates due to the vibration levels. And the higher the vibration, the higher the estimated standard deviations associated is delivered. Examination of the box plot in Figure 6 suggests that the measured flow rate at no vibration has huge variation compared to the medium and high vibration levels. In fact a regression analysis based on vibration only at the three levels shows that there is no significant difference for the medium and high vibrations, but the latter are significantly different from the no vibration scheme.

\section{Discussion}

The analysis of insulin delivery in CSII has been shown to be a very challenging task. Many factors have to be taken into account.

Firstly, the analysis cannot be performed on just one variable, whilst ignoring the others. The flow rate behaves differently depending on the insulin type, and vice-versa. The flow rate of $0.1 \mathrm{U} / \mathrm{hr}$ were found to be the most consistent in terms of the corresponding standard deviations. In fact, the experiments in week 2, which are

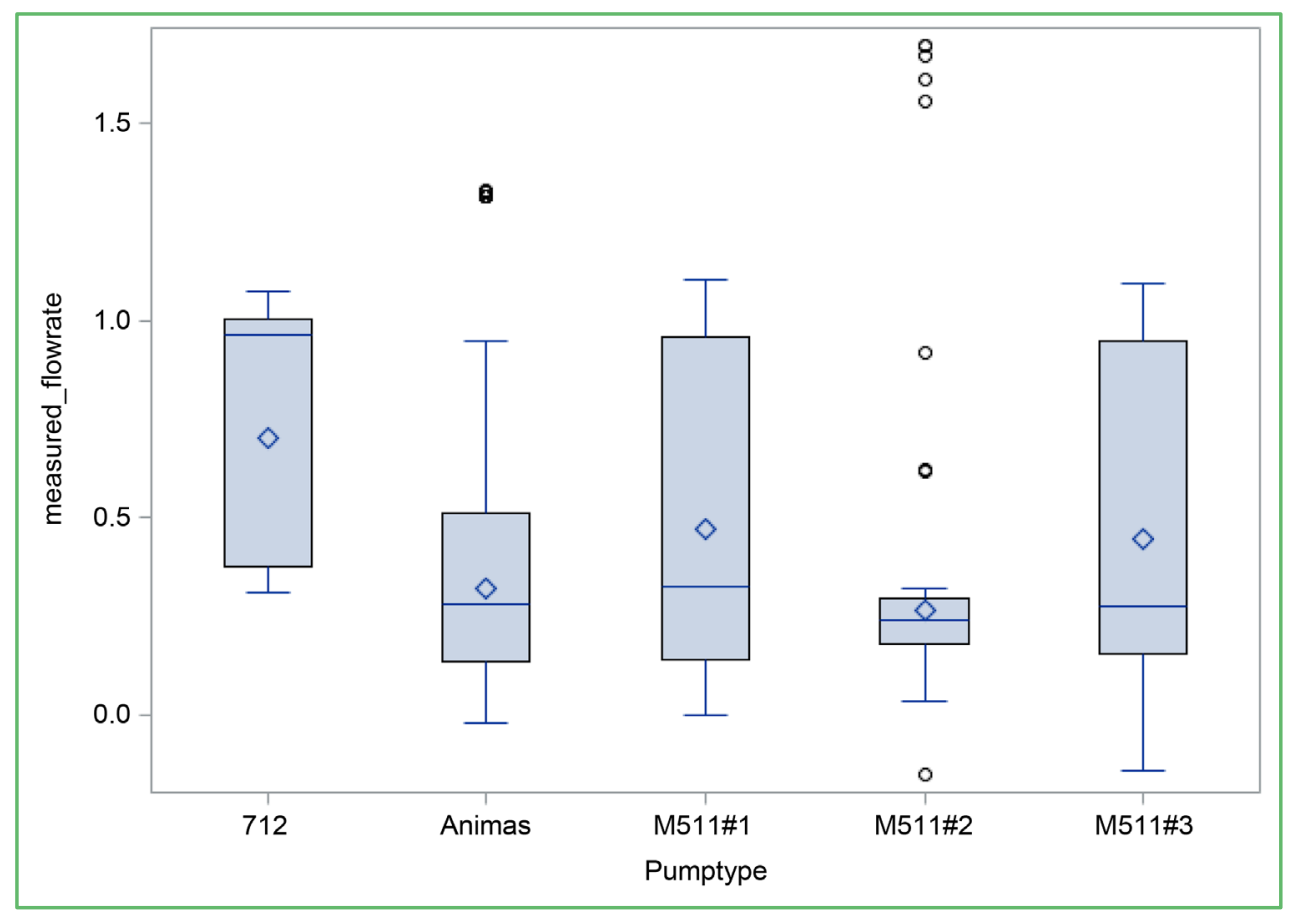

Figure 5. Distribution of the measured flow rate (U/hr) by pump types.

Table 6. Measured flow rate (U/hr) by the vibration level (RPM).

\begin{tabular}{ccc}
\hline Vibration & Mean measured flow rate & Standard deviation \\
\hline 0 & 0.280 & 0.297 \\
100 & 0.564 & 0.392 \\
200 & 0.516 & 0.430 \\
\hline
\end{tabular}




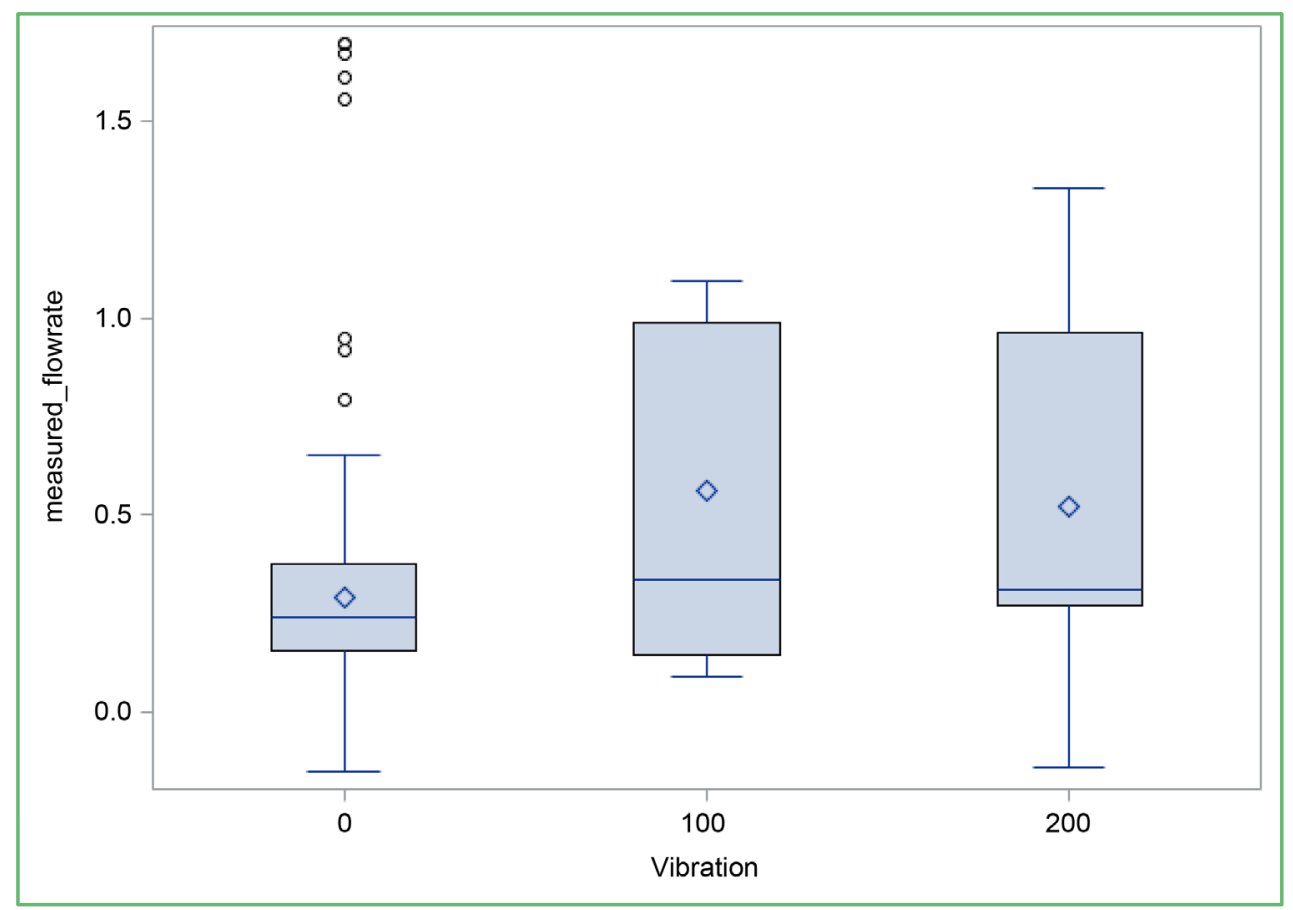

Figure 6. Effect of measured flow rate (U/hr) by vibration level (RPM).

at low flow rate with medium vibration, yielded the smallest standard deviation. So pump type M511 operates as expected with low flow rate and no vibration. It also appears that the pump types M511 and M712 perform better with Humalog. The vibration could be another factor to be considered with Humalog, as Humalog with vibration at level 3 yielded the most variation in insulin delivery.

Secondly, the insulin type used is an important factor as Apidra usage in weeks 4, and 5 gave higher mean value of insulin, whereas Novolog appeared to be the most stable, with the smallest associated variation. Another potential bias is the selection of pump type: pump type M712 has higher return of insulin than Animas and M511: further experiments are need for the comparison between Animas and M511.

Comparing the data from week 1 flow 1 , and week 2 flow 1 , we find that there is significant difference in insulin delivery due to insulin type, or pump type, or vibration or any combinations of those 3 . Apridra yields higher flow than Novolog, and finally Humalog.

We assumed that there is no significant difference in the pumps types M511. However, comparing the data obtained from week 4 in their first two experiments, a test of trend with respect to flow rate should be performed. If the assumption of no trend with flow rate is to be rejected, one should consider the parameters in analyzing the pumps of type M-511. Moreover, instead of flow rate, a further study could be done to check that assumption, taking into account the insulin types: Humalog, Apidra or Novolog. The higher vibration level with the use of M712 leads to higher volume of insulin than the lower vibration level and the use of M511.

In summary, the higher the preset flow rate, the higher the delivered insulin volume. At low flow rate and low variation level, Humalog is recommended. Similarly, at low flow rate and high vibration level, Apidra is recommended. Novolog works significantly well at low vibration level, but for high flow rate or medium/high vibration, it is not recommended.

\section{Conclusions}

Insulin delivery via CSII is a complex task in which the quality and reliability of the system must be tested with a model including different parameters, such as pump type, insulin type, activity level, etc. Because of that, the simple regression model will not be adequate. In this study, we were interested in the discriminations between components of the insulin delivery hypothesis using a linear response function, after careful selection of the experimental design and characteristic components. The design has allowed us to minimize the total variation and the number of experimental simulation runs to consider while keeping all significant factors. Several model com- 
binations of factors have been studied. Our research has shown that the insulin delivery in infants is subjected to several factors such as pump types, flow rate, insulin types. It is apparent that either frequent recalibration of the flow meters is necessary or a process for correcting "off-calibration" Sensirion flow rate data needs to be developed. All flow meters showed signs of calibration shifts to occur to some degree. The most relevant elements of our simulated study are the following:

1) If one aims to control the insulin delivery at low levels of flow rate and vibration, then the insulin types Apidra and Novolog should be used. High vibration level may be detrimental in the CSII delivery regardless of the insulin types. At low levels of flow rates and vibration, the pump type Animas gives a better control of the insulin delivery.

2) At high vibration level, Novolog and Humologare are preferred to Apidra.

Other factors such as bubble formation should also be considered in more details in future studies. The number of replicates could be an issue in this report as the power analysis would lead to better predictions. The statistical computations show that the process is affected by insulin and pump types, vibration level and flow rates.

\section{Acknowledgements}

The authors are very grateful for support from the Department of Pediatrics at East Virginia Medical School and the Office of Research at Old Dominion University.

\section{References}

[1] Pickup, J. and Keen, H. (2002) Continuous Subcutaneous Insulin Infusion at 25 Years: Evidence Base for the Expanding Use of Insulin Pump Therapy in Type 1 Diabetes. Diabetes Care, 25, 593-598. http://dx.doi.org/10.2337/diacare.25.3.593

[2] Blair, J., Gregory, J.W. and Peak, M. (2012) Insulin Delivery by Multiple Daily Injections or Continuous Subcutaneous Insulin Infusion in Childhood: Addressing the Evidence Gap. Practical Diabetes, 29, 47-48. http://dx.doi.org/10.1002/pdi.1656

[3] Shatilin, S. and Phillip, M. (2008) The Use of Insulin Pump Therapy in the Pediatric Age Group. Hormone Research, 70, 14-21. http://dx.doi.org/10.1159/000129673

[4] Rossetti, P., Porcellati, F., Perriello, C.G., Torlone, E. and Bolli, G.B. (2008) Superiority of Insulin Analogues versus Human Insulin in the Treatment of Diabetes Mellitus. Archives of Physiology and Biochemistry, 114, 3-14. http://dx.doi.org/10.1080/13813450801900777

[5] Cryer, P.E. (2008) The Barrier of Hypoglycemia in Diabetes. Diabetes, 57, 3169-3176. http://dx.doi.org/10.2337/db08-1084

[6] Weissberg-Benchell, J., Goodman, S.S., Lomaglio, J.A. and Zebracki, K. (2007) The Use of Continuous Subcutaneous Insulin Infusion (CSII): Parental and Professional Perceptions of Self-Care Mastery and Autonomy in Children and Adolescents. Journal of Pediatric Psychology, 32, 1196-1202. http://dx.doi.org/10.1093/jpepsy/jsm050

[7] Wolpert, H.A., Faradji, R.N., Bonner, W.S. and Lipes, M.A. (2002) Metabolic Decompensation in Pump Users Due to Lispro Insulin Precipitation. BMJ, 324, 1253. http://dx.doi.org/10.1136/bmj.324.7348.1253

[8] Einhorn, D. (2004) Advances in Diabetes for the Millennium: Insulin Treatment and Glucose Monitoring. Medscape General Medicine, 6, 8.

[9] Poulsen, C., Langkjaer, L. and Worsoe, C. (2005) Precipitation of Insulin Products Used for Continuous Subcutaneous Insulin Infusion. Diabetes Technology \& Therapeutics, 7, 142-150. http://dx.doi.org/10.1089/dia.2005.7.142

[10] Demuren, A. and Doane, S. (2007) A Study of Insulin Occlusion Using the Medtronic Paradigm 511 Insulin Pump. ODU Research Foundation Project Report.

[11] Kerr, D., Morton, J., Whatley-Smith, C., Everett, J. and Begley, J.P. (2003) Laboratory-Based Non-Clinical Comparison of Occlusion Rates Using Three Rapid-Acting Insulin Analogs in Continuous Subcutaneous Insulin Infusion Catheters Using Low Flow Rates. Journal of Diabetes Science and Technology, 2, 450-455. http://dx.doi.org/10.1177/193229680800200314

[12] Wang, B., Demuren, A., Gyuricsko, E. and Hu, H. (2011) An Experimental Study of Pulsed Micro-Flows Pertinent to Continuous Subcutaneous Insulin Infusion Therapy. Experiments in Fluids, 51, 65-74. http://dx.doi.org/10.1007/s00348-010-1033-7

[13] Hedman, C.A., Lindstrom, T. and Arnqvist, H.J. (2001) Direct Comparison of Insulin Lispro and Aspart Shows Small Differences in Plasma Insulin Profiles after Subcutaneous Injection in Type 1 Diabetes. Diabetes Care, 24, 1120-1121. http://dx.doi.org/10.2337/diacare.24.6.1120 
[14] von Mach, M.A., Brinkmann, C., Hansen, T., Weilemann, L.S. and Beyer, J. (2002) Differences in Pharmacokinetics and Pharmacodynamics of Insulin Lispro and Aspart in Healthy Volunteers. Experimental and Clinical Endocrinology \& Diabetes, 110, 416-419. http://dx.doi.org/10.1055/s-2002-36428

[15] Callow, M.J., Dudoit, S., Gong, E.L., Speed, T.P. and Rubin, E.M. (2000) Microarray Expression Profiling Identifies Genes with Altered Expression in HDL-Deficient Mice. Genome Research, 10, 2022-2029. http://dx.doi.org/10.1101/gr.10.12.2022

[16] Cui, X., Hwang, J.T.G., Qiu, J., Blades, N.J. and Churchill, G.A. (2005) Improved Statistical Tests for Differential Gene Expression by Shrinking Variance Components Estimates. Biostatistics, 6, 59-75. http://dx.doi.org/10.1093/biostatistics/kxh018

[17] Cox, D.R. and Reid, N. (2000) The Theory of the Design of Experiments. Chapman \& Hall.

[18] Montgomery, D.C. (2009) Design and Analysis of Experiments. Wiley\& Sons.

[19] De Mets, D.L. (2002) Clinical Trials in the New Millennium. Statistics in Medicine, 21, 2779-2787. http://dx.doi.org/10.1002/sim.1281

\section{Submit or recommend next manuscript to SCIRP and we will provide best service for you:}

Accepting pre-submission inquiries through Email, Facebook, LinkedIn, Twitter, etc. A wide selection of journals (inclusive of 9 subjects, more than 200 journals)

Providing 24-hour high-quality service

User-friendly online submission system

Fair and swift peer-review system

Efficient typesetting and proofreading procedure

Display of the result of downloads and visits, as well as the number of cited articles

Maximum dissemination of your research work

Submit your manuscript at: http://papersubmission.scirp.org/ 